\title{
Condylar Resorption After Orthognathic Surgery
}

\author{
Constantinus Politis, PhD, ${ }^{* \dagger}$ Gaétan Van De Vyvere, MD, ${ }^{\ddagger}$ and Jimoh Olubanwo Agbaje, PhD*†
}

\begin{abstract}
The aim was to postoperatively evaluate a conservative treatment approach to bilateral condylar resorption after orthognathic surgery. A retrospective study was carried out on 730 consecutive patients undergoing sagittal split osteotomy, 2013 to 2016. The mean follow-up period was 2.29 years. Clinical and radiographic findings of patients with postoperative bilateral condylar resorption were searched. Syndromic patients and patients with juvenile rheumatoid arthritis were excluded from this study. Of the 730 patients, $6(0.82 \%)$ required treatments because of bilateral postoperative condylar resorption but had no surgery at the temporomandibular joint (TMJ). Five patients with TMJ symptoms because of postoperative condylar resorption were managed with conservative treatment. About 2 of the 6 patients were successfully retreated with orthognathic surgery in the upper jaw to close the open bite. The TMJ symptoms can successfully be managed with conservative therapy, whereas skeletal relapse can be retreated with orthognathic surgery in the upper jaw, depending on the amount of overjet. Patient undergoing orthognathic surgery may develop bilateral condylar resorption though the frequency is $<1 \%$, most of these patients can be managed conservatively.
\end{abstract}

Key Words: Condylar remodeling, condylar resorption, internal derangement, osteoarthrosis, relapse, sagittal split osteotomy

(J Craniofac Surg 2018;00: 00-00)

Te emporomandibular disorders (TMDs) are a complex and poorly understood set of conditions that cause pain and dysfunction in the temporomandibular joint (TMJ) and the muscles that control jaw movement. ${ }^{1}$ Varying causes have been associated with TMDs, among them are injury to jaw, the joint, or the muscles of head and neck, malocclusion, and oral behaviors such as grinding or clenching of teeth. ${ }^{2}$

Orthognathic surgery occurs with a spectrum of changes, including condylar remodeling, internal derangement, condylar resorption, osteoarthrosis, ankylosis, or no detectable changes at the joint structures at all. Patients with pre-existing TMDs can become better or worse after surgery. ${ }^{3,4}$ Patients without pre-existing TMJ problems

From the *OMFS-IMPATH Research Group, Department of Imaging and Pathology, Faculty of Medicine, Katholieke Universiteit Leuven; †Department of Oral and Maxillofacial Surgery, University Hospitals Leuven, Leuven; and $\ddagger$ Department of Oral and Maxillofacial Surgery, OLV Ziekenhuis, Aalst, Belgium.

Received April 25, 2018.

Accepted for publication June 16, 2018.

Address correspondence and reprint requests to Constantinus Politis, Jimoh Olubanwo Agbaje, Department of Oral and Maxillofacial Surgery, University Hospitals of Leuven, Kapucijnenvoer 33, 3000 Leuven, Belgium; E-mail: c.politis@village.uunet.be

The authors report no conflicts of interest.

Copyright (1) 2018 by Mutaz B. Habal, MD

ISSN: $1049-2275$

DOI: $10.1097 /$ SCS.0000000000004837 can also develop postoperative TMJ-related symptoms and even condylar resorption. 5

In a systematic review of the available literature between 1980 and 2008, Jerjes et $\mathrm{al}^{6}$ (2011) concluded that TMJ symptoms present before surgery were significantly reduced after orthognathic treatment of the lower jaw. They also concluded that a percentage of dysgnathic patients who were preoperatively asymptomatic may develop TMD after surgery, but this risk is low.

Condylar resorption is a feared complication of orthognathic surgery, causing relapse and alteration of results in orthognathic surgery (see Hoppenreijs ${ }^{7}$ for a recent review), characterized by radiographic condylar changes and the relapse of an open bite. Radiologic signs of condylar resorption also can be present in juvenile osteoarthritis and osteoarthrosis, and the diagnosis cannot be made on imaging alone. In addition, a postoperative open bite after orthognathic surgery can result from a multitude of causes unrelated to condylar resorption, which can cause confusion about the diagnosis. Regarding treatment, European centers tend to favor conservative approaches whereas other centers favor alloplastic joint replacements, ${ }^{8}$ even in well-functioning joints.

Condylar remodeling is the physiologic adaptation of the condylar structures, such as cartilage and bone, to the stresses exerted by orthognathic surgery and occurs in all condyles to a certain degree, without producing clinical signs or symptoms of temporomandibular disorders. Internal derangements imply that disc disorders were present before orthognathic surgery or appeared after orthognathic surgery. Complete disc dislocation or disc perforation forces the condyle to function close to the fossa surface, sometimes leading to osteoarthrotic changes. These changes may produce clinical signs of pain, joint sounds, limited opening of the mouth, and a class II malocclusion depending on the loss of condylar and fossa bony height. Rarely, an open bite will appear. The primary outcome of this study was to assess the frequency of condylar resorption from orthognathic patients who underwent sagittal split osteotomies from January 2013 to December 2016 in the University Hospitals of Leuven. The secondary outcome was the conservative mode of these patients' management.

The aim of the present study was to evaluate a conservative treatment approach toward postoperative bilateral condylar resorption after orthognathic surgery.

The clinical rationale of this study is to show that patient undergoing orthognathic surgery may develop condylar resorption (though the frequency is $<1 \%$ of patients) and to assess the role of concervative management in such patients.

\section{METHODS}

\section{Patients}

The files of orthognathic patients who underwent sagittal split osteotomies (with or without simultaneous Le Fort I osteotomy and/ or genioplasty) from January 2013 to December 2016 in the University Hospitals of Leuven (Belgium) were selected for retrospective analysis. The analysis included clinical data, panoramic and cephalometric radiographs, and, when available, cone-beam computed tomography (CBCT) data. The search focused on patient files that described bilateral condylar resorption with an open bite 


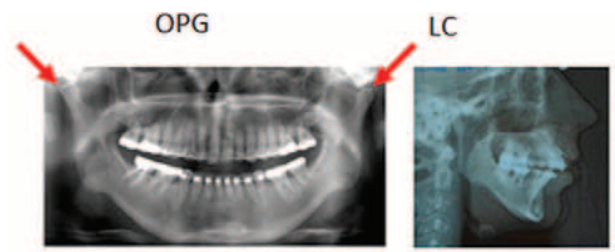

CP

A
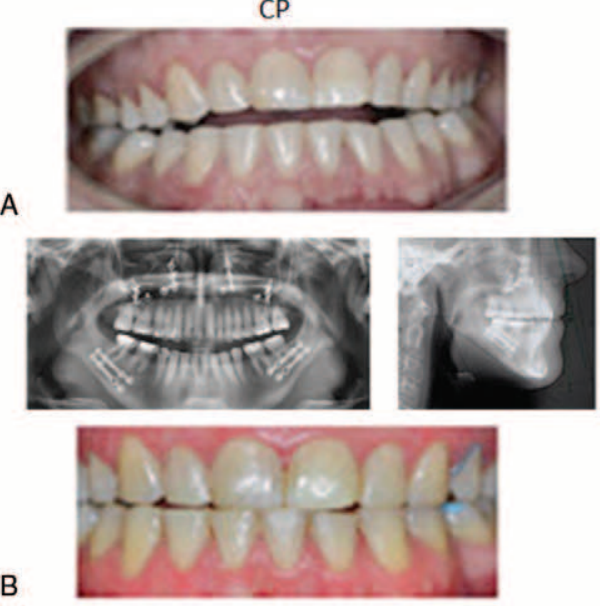

B

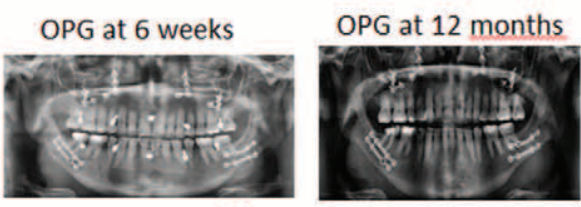

CP

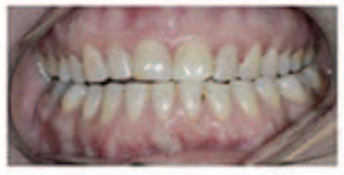

B

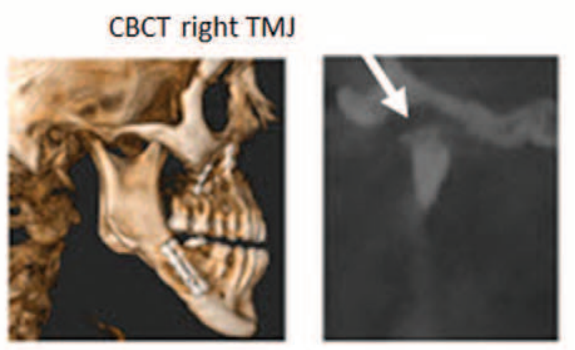

CBCT left TMJ
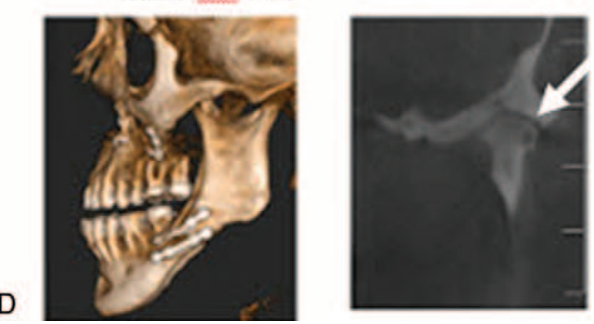

FIGURE 1. Panoramic radiograph, lateral cephalogram, cone-beam computed tomography $(\mathrm{CBCT})$, and clinical frontal photograph of patient 1. (A) Preoperative panoramic radiograph (OPG), lateral cephalogram (LC), and clinical frontal photograph (CP) depicting anterior open bite malocclusion. The panoramic radiograph shows well-developed and symmetric condyles with normal ascendic ramus height (red arrow). (B) Six-month postoperative panoramic radiograph, lateral cephalogram, and clinical frontal photograph depicting complete closure of the preoperative anterior open bite. The after orthognathic surgery. Excluded studies involved patients with congenital syndromes and patients with juvenile rheumatoid arthritis. The study was performed in accordance with Helsinki Declaration II. The study protocol was approved by the ethics committee of UZ Leuven S55873. All procedures were fully explained to all participants, who provided written informed consent.

Bilateral condylar resorption in this study is defined as a significant relapse after a mandibular advancement with the occurrence of an anterior open bite together with significant bilateral radiographic morphologic changes, decreased condylar volume, and decreased ramus height, with or without clinical TMJ symptoms of pain, limited mouth opening, or joint sounds. According to Proffit et al, ${ }^{9}$ occlusal changes (eg, relapse) of $<2 \mathrm{~mm}$ are considered to be within the range of error and clinically insignificant; occlusal changes of 2 to $4 \mathrm{~mm}$ are considered potentially clinically significant; and occlusal changes $>4 \mathrm{~mm}$ are viewed as clinically highly significant and often beyond the range of orthodontic compensation.

\section{Operative Technique}

All patients were operated by the same surgical team. The TMJ symptoms were not considered a contraindication to orthognathic surgery. Otherwise, the stance was to proceed with orthognathic surgery and evaluate residual symptoms at the 12-month followup visit.

The Le Fort I osteotomy was performed as described by Bell et al. ${ }^{10}$ All patients had fixed orthodontic appliances with attached surgical hooks to allow for intermaxillary fixation of the teeth into an occlusion set by the intermediate and final surgical splints. Intermaxillary fixation intraoperatively involved metal wires during the intraoperative phase and orthodontic elastics in the postoperative phase. The osteotomy sites were stabilized with KLS-Martin miniosteosynthesis plates (KLS Martin GmbH, Freiburg, Germany).

After mobilization and repositioning of the maxilla, 4 ( 2 on each side) L-formed mini-plates were used to fix the maxilla to its new position, based on the intermediate surgical splint. In multisegmental Le Fort I osteotomies, more than 4 miniplates were used for rigid fixation.

Bone grafts were interposed in all extrusions with a visible vertical gap, in all maxillary advancements $>4 \mathrm{~mm}$, and in all multisegmental maxillary osteotomies. Sagittal split osteotomies were performed according to the Epker-Hunsuck modification and stabilized with 2 monocortical miniplates at each side, according to Tulasne and Schendel. ${ }^{11-14}$ No intermaxillary fixation was used, except for light elastics on surgical hooks to guide the patient into the correct occlusion during jaw movements. All unerupted third molars were removed at least 6 months before osteotomy surgery.

\section{RESULTS}

Six patients $(0.82 \%), 5$ females and 1 male, with mean age of 20 years with age ranging from 16 to 25 years, with bilateral condylar resorption were identified according to the definition used from a sample of 730 orthognathic surgery patients in the period

panoramic radiograph shows well-developed and symmetric condyles with normal ascendic ramus height. (C) Panoramic radiographs at 6 weeks and 12 months postoperative after the redo bilateral sagittal split osteotomy with a bone graft to close the open bite, which recurred during the pregnancy of the patient after the first orthognathic procedure. Clinical picture demonstrating a stable result at 12 months after the redo procedure. Although the occlusion is far from perfect, the midlines were preserved as was end-to-end incisor contact. The patient refused renewed orthodontic treatment. (D) CBCT of the right temporomandibular joint (TMJ) depicting a posterior slope of the condylar process, osteoarthrotic changes, and a well-preserved joint space; CBCT of the left TMJ depicting a posterior slope of the condylar process, osteoarthrotic changes, and a severely diminished joint space (white arrow). 
2013 to 2016. One patient developed bilateral condylar resorption during pregnancy within 12 months after orthognathic surgery. All patients could be managed with conservative therapy, so no alloplastic joint reconstruction was done.

\section{Clinical Presentations}

Patient 1 underwent bimaxillary surgery at age 25 (Fig. 1A-B). The patient was without complaints before surgery, but at 7 months postoperative, the patient was pregnant and subsequently developed a midline shift of the jaws, which eventually resulted in a symmetric total open bite. After pregnancy, the panoramic radiograph at 1.6 years postoperative showed a bilateral condylar resorption. A redo bilateral sagittal split osteotomy (BSSO) was done at 3 years postoperative, with a bone graft interposition at the BSSO gap. After 1 year and 8 months (5 years after the initial bimaxillary procedure), the occlusion (photograph), panoramic radiograph, and CBCT showed stabilization of the condylar resorption (Fig. 1C-D). The CBCT showed loss of joint space at the left joint and loss of linear demarcation of bone at the fossa level both at the left and right joints, indicative of osteoarthrosis. The patient had no pain or joint sounds, maximal mouth opening was limited to $31 \mathrm{~mm}$, and lateral excursions of the lower jaw were possible up to $7 \mathrm{~mm}$ on each side.

Patient 2 had bimaxillary surgery at age 17 and developed a class II open bite due to condylar resorption 1 year after surgery. The panoramic radiograph and the clinical intraoral picture were taken at 2 years postoperative. Patient was placed on minocycline $50 \mathrm{mg}$ per day for 6 months after which a corrective 3-piece Le Fort I osteotomy was done to correct the malocclusion. Occlusion remained stable at the 2-year postoperative follow-up (Fig. 2). During the entire process (pre- and postcorrective 3-piece Le Fort I osteotomy and follow-up period), the patient did not develop TMJ complaints. At the last follow-up, 4 years after the initial bimaxillary surgery, the maximal mouth opening was $43 \mathrm{~mm}$, without joint pain or joint sounds. The CBCT showed a total loss of both condyles without disappearance of the joint space (white arrow, Fig. 2D).

Patient 3 is a 18 -year-old female, she had severe retrognathia (Fig. 3A) and underwent a counter clockwise rotation of the lower jaw and genioplasty together with a posterior extrusion of the maxilla with bone graft at age 15 . The initial results, both clinical and on the lateral head radiograph (Fig. 3B) were satisfactory. Three months postoperatively, she developed a class II open bite, and the panoramic radiograph and CBCT showed bilateral condylar resorption without TMJ pain, but a locked TMJ at the left side. The CBCT showed changes only at the condyle and none at the fossa. As of last check-up ( 2 years postoperatively), patient is pain free, with good mouth opening of up to $45 \mathrm{~mm}$.

Patient 4 is a 16-year-old female who underwent a BSSO surgery. Preoperatively, she had no TMJ complaints. Within 2 months postoperatively, however, she developed TMJ complaints with restricted interincisal opening to a maximum of $35 \mathrm{~mm}$, accompanied by pain and crepitation at the left joint. Splint therapy and physical therapy were initiated. Progressively, the right joint also became painful. An open bite became apparent at 1 year postoperative, after the joint complaints. The x-rays show the situation at 1.5 years postoperative (Fig. 4A). Three years after the initial BSSO surgery, the symmetrical open bite has stabilized (Fig. 4B), and the interincisal opening had increased to $45 \mathrm{~mm}$. The CBCT at 3 years postoperative (Fig. 4B) showed total loss of the condylar volume at both sides, but with a bilaterally well-preserved joint space. At age 19, the patient was free of pain, and she continued wearing a stabilizing occlusal splint and very rarely had complaints at the left joint. No corrective surgery was initiated to correct the postoperative malocclusion.
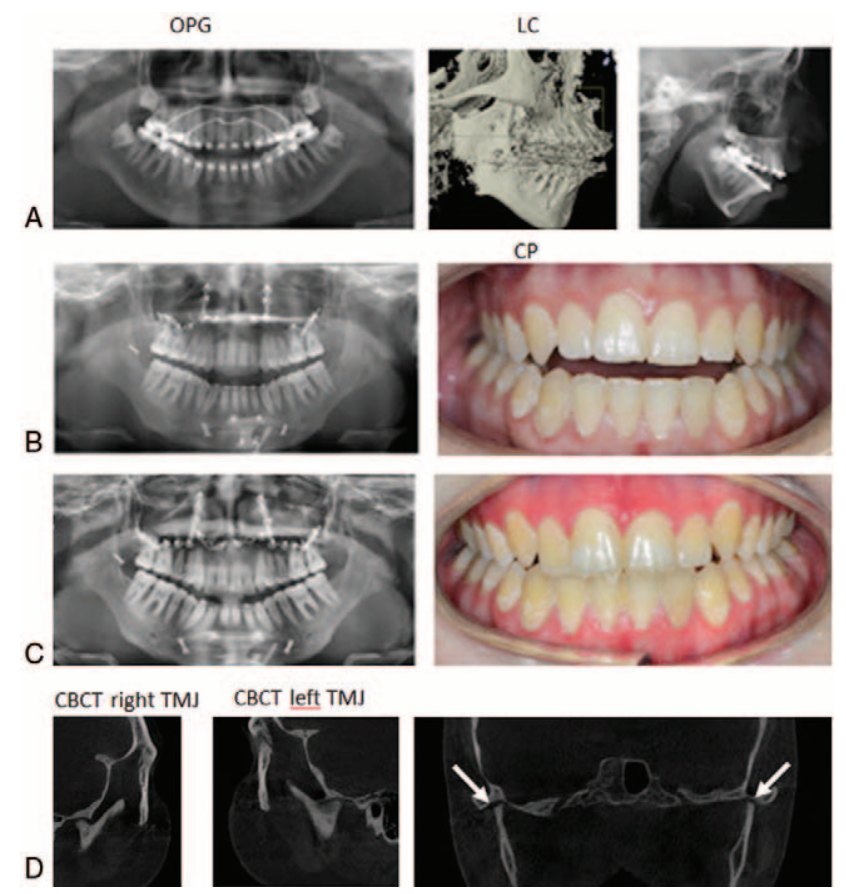

FIGURE 2. Panoramic radiograph, lateral cephalogram, cone-beam computed tomography (CBCT), and clinical frontal photograph of patient 2. (A) Panoramic radiograph, $\mathrm{CBCT}$, and cephalometric radiograph at age 16.5 years before the first orthognathic procedure to correct severe retrognathia and open bite. The radiographs depict several risk factors that could lead to postoperative condylar resorption: small condyles, posterior sloping of the condylar process, and an antegonal notch at the mandibular angle. (B) Status after relapse of the open bite due to bilateral condylar resorption after the first orthognathic surgery. Osteosynthesis plates in the lower jaw have been removed. Clinical picture (right image) shows poor oral hygiene and an anterior open bite extending into the premolar area. No class II development due to compensatory orthodontic inclination of the lower incisors. (C) Patient status 1 year after a corrective 3piece Le Fort I osteotomy. The malocclusion has been corrected and has remained stable (right image) in spite of "total loss" of condylar volume, as can be seen in the panoramic radiograph. (D) Patient CBCT at 1-year postoperative after the 3-piece Le Fort I osteotomy, illustrating the complete disappearance of condylar volume. Enlargement of the coronoid process. Narrowing of the joint space. In spite of these radiographic changes, this patient at no time developed temporomandibular joint symptoms, and the occlusion remained stable after the second orthognathic surgery (3-piece Le Fort I osteotomy).

Patient 5 is a 23-year-old female who developed a bilateral condylar resorption 3 years after a bimaxillary osteotomy. Intraorally, the patient presented with a class I open bite and narrow maxillae (Fig. 5). The maximum interincisal mouth opening was restricted to $30 \mathrm{~mm}$, without joint sounds or pain upon palpation of the joints. Patient was managed conservatively, the maximal opening of the mouth remained limited to $34 \mathrm{~mm}$, but patient remained pain free.

Patient 6 is a male patient, he had a BSSO at age 21 . He had no TMJ complaints before orthognathic surgery. Six months after BSSO, crepitations and pain developed in the right TMJ. Arthroscopic lysis and lavage of the affected right joint showed inflammation both at the synovial side and the bony side of the fossa. Three years postoperatively, the patient is entirely pain free, without joint sounds and an unlimited opening of the mouth up to $47 \mathrm{~mm}$.

\section{DISCUSSION}

We observed an incidence of $0.82 \%$ of bilateral condylar resorption among orthographic patients treated in our center. Though this incidence is slightly less than what is reported in the literature with 


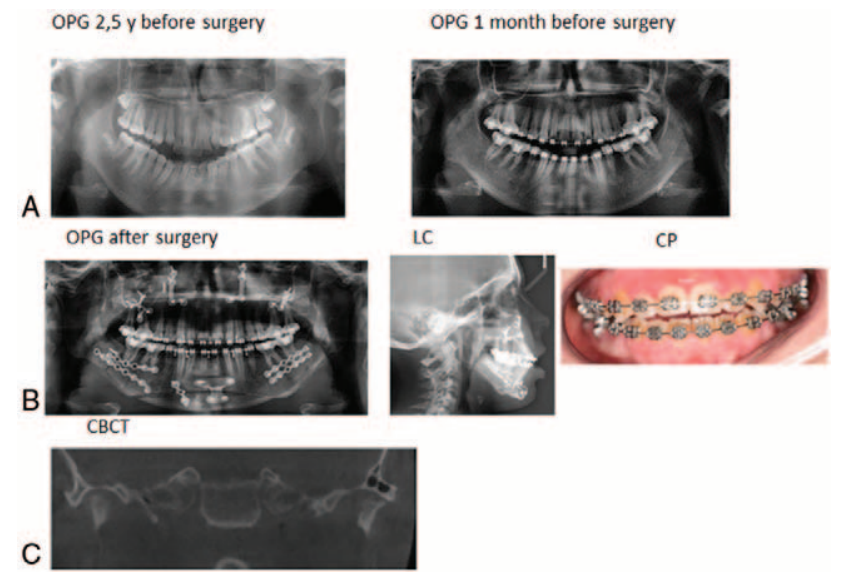

FIGURE 3. Panoramic radiograph, lateral cephalogram, cone-beam computed tomography (CBCT), and clinical frontal photograph of patient 3. (A) Panoramic radiograph 2.5 years before orthognathic surgery at age 12.5 years, depicting a normal ramus height and condylar volume; panoramic radiograph 1 month before orthognathic surgery at age 15 depicting a reduction in condylar volume at both the left and right joints, development of a posteriorly sloping condylar process, and development of an antegonal notch. These radiographic

developments constitute risk factors for possible development of postoperative condylar resorption. (B) Panoramic radiograph and lateral cephalometric radiograph 6 weeks postoperative. A counter clockwise rotation of the maxillamandibular complex was achieved with bone interposition and a genioplasty. No relapse tendency was seen at 6 weeks postoperative. At 3 months postoperative, a clinically obvious relapse occurred of the open bite together with a class II malocclusion. (C) The CBCT at 3 months postoperative, demonstrating a well-preserved bony contour at the fossa both at the right and left sides, and a bilaterally well preserved joint space, but marked contour changes and flattening of the condyles, more prominently at the left side.

the reported incidence of condylar resorption after orthognathic surgery ranging from $1 \%$ to $31 \% .^{15-17}$ In the literature both unilateral and bilateral patients of condylar resorption are reported. The reported incidence is mainly depending on the definition used, ${ }^{18,19}$ or depending on the variations in the populations, knowing that postoperative bilateral condylar resorption is mainly or
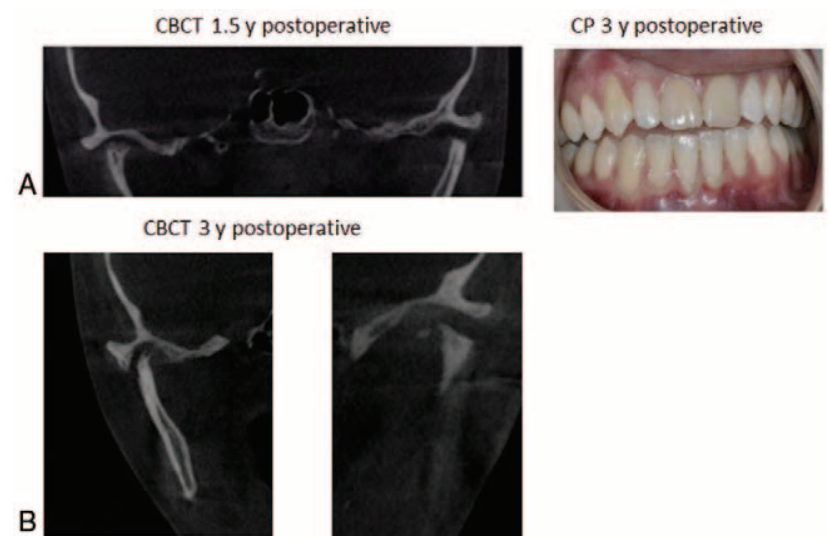

FIGURE 4. Cone-beam computed tomography (СВCT) and clinical fronta photograph of patient 4 . (A) CBCT at 1.5 years postoperative. The contour of the fossa was well preserved, as was the joint space. However, the condylar resorption was extreme at both right and left sides. Clinical picture of the postoperative open bite in the patient at 3 years postoperative, extending up to the molars. (B) CBCT at 3 years postbilateral sagittal split osteotomy demonstrating a total loss of condylar volume at both joints; however, without loss of bony contour at the fossa side and with a well-preserved joint space (left image: left temporomandibular joint [TMJ]; right image: right TMJ).
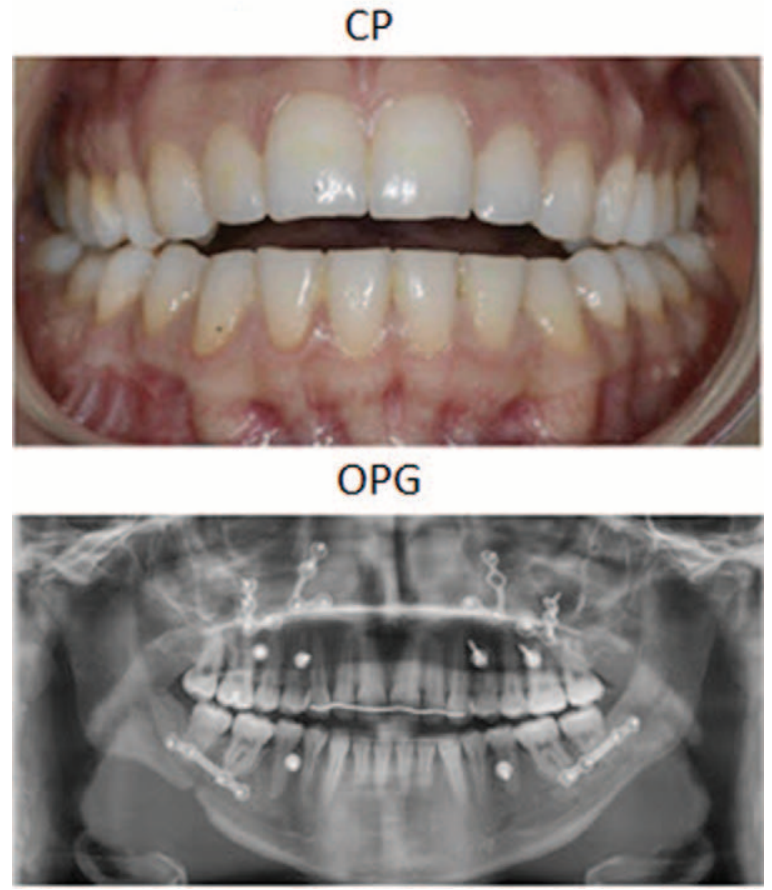

FIGURE 5. Clinical photograph and panoramic radiograph of patient 5. Clinical picture of a postoperatively developed anterior open bite extending to the premolar area. The panoramic radiograph demonstrates signs of condylar resorption more pronounced at the right than at the left side, and yet the dental midline of the lower jaw was not shifted to the most affected side.

exclusively a complication of sagittal split advancement surgery and is rarely seen in populations with a class III malocclusion. ${ }^{20}$

Between 1989 and 2017, more than 3500 orthognathic patients have been followed up by the first author (CP). The first signs of condylar resorption are invariably clinical with the occurrence of significant relapse and symmetrical open bite, sometimes preceded by transient clinical TMJ pain in 1 or 2 joints. Posnick ${ }^{21}$ found similar signs, mentioning the 3-month postoperative period as the initial clinical period for diagnosing condylar resorption. Posnick ${ }^{21}$ used the following mandibular and occlusal findings as part of the radiographic diagnosis: change in the shape of the condylar heads (ie, flattening and thinning); decrease in condylar height; loss of overall posterior facial height; mandibular retropositioning with clockwise rotation (high angle); and angle class II. For most authors, the radiologic signs of resorption follow the clinical findings and become apparent at the radiographic checkpoint of 6 months or even later at 12 months, ${ }^{22}$ probably because of the radiographic protocol, which limits full radiographic data (panoramic radiograph, lateral cephalogram, and $\mathrm{CBCT}$ ) at 1 week, 6 weeks, 6 months, 12 months, and 24 months postoperatively. If pain and TMJ sounds occur in the first few months after orthognathic surgery, they should raise suspicion of condylar changes occurring in the following months. Panoramic radiographs, serial lateral cephalograms, CBCT, technetium isotope bone scan, and positron emission tomography-magnetic resonance imaging may be used to establish the diagnosis. However, the findings of the current retrospective study in 730 patients suggest that condylar resorption at the end of the process leaves the patient without TMJ pain, probably at the expense of loss of significant ramus height. This distinction clinically separates bilateral condylar resorption as defined in this study from condylar remodeling and from internal derangements occurring without severe relapse. 
Both nonsurgical and surgical risk factors have been implicated in condylar resorption but without conclusive evidence. Identified risk factors include demographic and biometric factors such as female, age range from 15 to 35 years, preoperative TMD, high mandibular plane angle with mandibular hypoplasia, and a small posterior-to-anterior facial height ratio. ${ }^{17,23-28}$ Also posterior inclined condylar neck has been considered another contributing factor, ${ }^{16,17,26}$ estrogen imbalance, nutritional status (dietary omega3 fatty acids, vitamin D), individual patient genetic susceptibility, bruxism, repetitive oral habits, displaced articular discs, adolescent idiopathic condylar resorption, reactive arthritis, and connective tissue and autoimmune diseases are risk factors. ${ }^{23,24,29-31}$ Surgical factors such as mandibular advancement, maxillary impaction, mandibular autorotation, and bimaxillary surgery can induce condylar resorption. The incidence of condylar resorption has been found to be significantly higher after bimaxillary osteotomy. ${ }^{7,17}$

The pathogenesis of condylar resorption remains unclear. One hypothesis correlates it with increased, abnormal joint loading and subsequent pressure resorption, and it may occur after orthodontics, orthognathic surgery, occlusal therapy, internal derangement, parafunction, trauma, and unstable occlusion. ${ }^{23,32}$ Another hypothesis is that the mechanism of condylar resorption is the same as that seen in avascular necrosis of the femoral head. Others believe that condylar resorption may be the result of loss of the normal remodeling capacity of the condyle caused by factors such as age, systemic illness, and hormones. ${ }^{32}$

Once the onset of condylar resorption has started, an active period of condylar changes during 12 to 24 months need to be expected, resulting in relative TMJ stability with usually a satisfactory range of motion of the mandible, and no significant TMJ limitations, but with a high angle anterior open bite malocclusion. Some discomfort during chewing usually responds well to splint therapy. Borstlap et $\mathrm{al}^{33}$ reported a patient satisfaction rate of $90 \%$ irrespective of the condylar changes. The results of the present review support this view. This finding has far-reaching treatment implications because allowing condylar changes to tamp out ultimately leaves only the residual malocclusion and loss of gained facial aesthetics as indications to reoperate.

In the present study, only 1 patient with bilateral condylar resorption evolved to osteoarthrosis with loss of joint space but with a satisfactory end result. In most of our patients with condylar resorption the end stage is characterized by skeletal relapse but little or no TMJ symptoms or mouth opening restrictions after conservative management. It may well be that the preservation of joint space, as we have seen in most patients with bilateral condylar resorption, is the cornerstone of this favorable outcome at the TMJ. On the contrary in osteoarthrosis, subchondral changes at the condyle affect the disc, and it may well be that the vertical collapse of the condyle as witnessed in condylar resorption allows the soft tissue contents to overcome the inflammatory stage without irreversible damage.

Condylar remodeling is the physiologic adaptation of the condylar structures, such as cartilage and bone, to the stresses exerted by orthognathic surgery and occurs in all condyles to a certain degree, without producing clinical signs or symptoms of temporomandibular disorders. ${ }^{34}$ Bilateral condylar resorption is a rare event after orthognathic surgery in predominantly young females with a presumptive risk profile. The main clinical picture is the appearance of an open bite due to skeletal relapse with apparent radiographic changes at the TMJs. Ultimately the joint function remains well preserved.

Although recent studies ${ }^{22,35}$ prove that some class II patients demonstrate a remodeling that exceeds the adaptive capacities of the stomatognathic system to retain the postoperative class I occlusion, we believe that this still does not qualify for the label of "condylar resorption" because this would qualify resorption as an excess of remodeling. Our belief is that this is not an accurate rendition of clinical reality: bilateral condylar resorption patients are a separate entity, clearly separated from physiologic remodeling, internal derangement with or without osteoarthrosis.

The clinical observations from the present study allow us to hypothesize 3 different healing mechanisms after orthognathic surgery which can occur independently in different patients: physiologic remodeling in most patients, allowing adaptive changes to maintain a stable occlusion and a stable joint; internal derangements with secondary or simultaneous degradation of the cartilaginous surfaces in the vicinity of the condylar surface and a possible evolution to osteoarthrosis from there once the cartilage has lost its protective function of the underlying bone; or bilateral condylar resorption affecting mainly the condylar process with rapid loss of the vertical structure of the condyle, while affecting the fibrocartilaginous surfaces of the joint less, possibly because of preservation of sufficient joint space allowing nutrition and lubrication of the remaining bilaminary zone and disc or disc remnants. Only if disc function is not preserved could this condition lead to osteoarthrosis.

The fact that in all patients of condylar resorption, a conservative approach was chosen may have been facilitated by regional organization of Belgian health care with short distances and a lowthreshold health care system with moderate financial burdens for patients in need of frequent visits to health care professionals (counseling, physical therapy, splint therapy) over a long period of time. At the same time, an alloplastic total joint prosthesis is poorly reimbursed, placing a heavy financial burden on the patient with bilateral condylar resorption should they opt for this surgical solution.

\section{Limitations}

This study is not free of limitations. The retrospective observational design is a concern. However, the long and strict follow-up of patients allow a decent evaluation of a conservative treatment approach.

\section{CONCLUSION}

Bilateral condylar resorption resulting in open bite is a rare complication of orthognathic surgery in the present retrospective study of 730 patients, occurring in $0.82 \%$ of patients. A bilateral condylar resorption patient is either young with severe retrognathia, high angle, and small condyles, or is a young woman who develops bilateral condylar resorption during pregnancy. Physiologic condylar remodeling and bilateral condylar resorption do not appear to be related stages of the same process. The end stage of bilateral condylar resorption is a patient with a symmetrical anterior open bite without TMJ complaints. In this study, no reoperation at the level of the TMJs seemed necessary.

\section{REFERENCES}

1. Romero-Reyes M, Uyanik JM. Orofacial pain management: current perspectives. J Pain Res 2014;7:99-115

2. Leketas M, Saferis V, Kubilius R, et al. Oral behaviors and parafunctions: comparison of temporomandibular dysfunction patients and controls. J Craniofac Surg 2017;28:1933-1938

3. Al-Riyami S, Moles DR, Cunningham SJ. Orthognathic treatment and temporomandibular disorders: a systematic review. Part 1. A new quality-assessment technique and analysis of study characteristics and classifications. Am J Orthod Dentofacial Orthop 2009;136:624.e1624.e 15

4. Jung HD, Kim SY, Park HS, et al. Orthognathic surgery and temporomandibular joint symptoms. Maxillofac Plast Reconstr Surg 2015;37:14

5. Pelo S, Saponaro G, De AP, et al. Effect of surgery first orthognathic approach on the temporomandibular joint. J Craniofac Surg 2018;29:671-675 
6. Jerjes W, Hamdoon Z, Abbas S, et al. Temporomandibular joint dysfunction in orthognathic surgery: systematic review. $\mathrm{Br} \mathrm{J} \mathrm{Oral}$ Maxillofac Surg 2011;49:S70

7. Hoppenreijs TJ, Maal T, Xi T. Evaluation of condylar resorption before and after orthognathic surgery. Semin Orthod 2013;19:106-115

8. Abela S, Tewson D, Prince S, et al. Total TMJ reconstruction in cases of advanced idiopathic condylysis. J Clin Orthod 2015;49:263-269

9. Proffit WR, Turvey TA, Phillips C. The hierarchy of stability and predictability in orthognathic surgery with rigid fixation: an update and extension. Head Face Med 2007;3:21

10. Bell WH, Mannai C, Luhr HG. Art and science of the Le Fort I down fracture. Int J Adult Orthodon Orthognath Surg 1988;3:23-52

11. Hunsuck EE. A modified intraoral sagittal splitting technic for correction of mandibular prognathism. J Oral Surg 1968;26:250-253

12. Tulasne JF, Schendel SA. Transoral placement of rigid fixation following sagittal ramus split osteotomy. J Oral Maxillofac Surg 1989;47:651-652

13. Epker BN. Modifications in the sagittal osteotomy of the mandible. J Oral Surg 1977;35:157-159

14. Mommaerts MY. Guidelines for patient-specific jawline definition with titanium implants in esthetic, deformity, and malformation surgery. Ann Maxillofac Surg 2016;6:287-291

15. De Clercq CA, Neyt LF, Mommaerts MY, et al. Condylar resorption in orthognathic surgery: a retrospective study. Int J Adult Orthodon Orthognath Surg 1994;9:233-240

16. Hwang SJ, Haers PE, Sailer HF. The role of a posteriorly inclined condylar neck in condylar resorption after orthognathic surgery. J Craniomaxillofac Surg 2000;28:85-90

17. Hoppenreijs TJ, Stoelinga PJ, Grace KL, et al. Long-term evaluation of patients with progressive condylar resorption following orthognathic surgery. Int J Oral Maxillofac Surg 1999;28:411-418

18. Dicker G, Van SP, Van SR, et al. Adaptation of jaw closing muscles after surgical mandibular advancement procedures in different vertical craniofacial types: a magnetic resonance imaging study. Oral Surg Oral Med Oral Pathol Oral Radiol Endod 2007;103:475-482

19. Papadaki ME, Tayebaty F, Kaban LB, et al. Condylar resorption. Oral Maxillofac Surg Clin North Am 2007;19:223-234

20. Kobayashi T, Izumi N, Kojima T, et al. Progressive condylar resorption after mandibular advancement. Br J Oral Maxillofac Surg 2012;50:176180

21. Posnick JC. Orthognathic Surgery - 2 Volume Set: Principles and Practice. London, UK: Elsevier; 2014:1783
22. Xi T, Schreurs R, van LB, et al. 3D analysis of condylar remodelling and skeletal relapse following bilateral sagittal split advancement osteotomies. J Craniomaxillofac Surg 2015;43:462-468

23. Arnett GW, Milam SB, Gottesman L. Progressive mandibular retrusionidiopathic condylar resorption. Part I. Am J Orthod Dentofacial Orthop 1996;110:8-15

24. Arnett GW, Milam SB, Gottesman L. Progressive mandibular retrusionidiopathic condylar resorption. Part II. Am J Orthod Dentofacial Orthop 1996;110:117-127

25. Huang YL, Pogrel MA, Kaban LB. Diagnosis and management of condylar resorption. J Oral Maxillofac Surg 1997;55:114-119

26. Hwang SJ, Haers PE, Zimmermann A, et al. Surgical risk factors for condylar resorption after orthognathic surgery. Oral Surg Oral Med Oral Pathol Oral Radiol Endod 2000;89:542-552

27. Kerstens HC, Tuinzing DB, Golding RP, et al. Condylar atrophy and osteoarthrosis after bimaxillary surgery. Oral Surg Oral Med Oral Pathol 1990;69:274-280

28. Moore KE, Gooris PJ, Stoelinga PJ. The contributing role of condylar resorption to skeletal relapse following mandibular advancement surgery: report of five cases. J Oral Maxillofac Surg 1991;49:448-460

29. Anonymous. Research on orthognathic surgery-an NIDR state-of-theart workshop. J Oral Surg 1975;33:907-920

30. Catherine Z, Breton P, Bouletreau P. Condylar resorption after orthognathic surgery: a systematic review. Rev Stomatol Chir Maxillofac Chir Orale 2016;117:3-10

31. Gill DS, El MM, Naini FB. Risk factors for post-orthognathic condylar resorption: a review. World J Orthod 2008;9:21-25

32. Gunson MJ, Arnett GW, Milam SB. Pathophysiology and pharmacologic control of osseous mandibular condylar resorption. J Oral Maxillofac Surg 2012;70:1918-1934

33. Borstlap WA, Stoelinga PJ, Hoppenreijs TJ, et al. Stabilisation of sagittal split advancement osteotomies with miniplates: a prospective, multicentre study with two-year follow-up. Part III-condylar remodelling and resorption. Int J Oral Maxillofac Surg 2004;33:649-655

34. Ha MH, Kim YI, Park SB, et al. Cone-beam computed tomographic evaluation of the condylar remodeling occurring after mandibular setback by bilateral sagittal split ramus osteotomy and rigid fixation. Korean J Orthod 2013;43:263-270

35. Xi T, Schreurs R, Heerink WJ, et al. A novel region-growing based semi-automatic segmentation protocol for three-dimensional condylar reconstruction using cone beam computed tomography (CBCT). PLoS One 2014;9:e111126 